\title{
Lessons of the month 2: Connecting the clues: limb swelling and pain
}

\author{
Authors: Timothy Prossor, ${ }^{A}$ Kokul Sriskandarajah, ${ }^{B}$ Frederick W Vonberg ${ }^{C}$ and Melanie Dani ${ }^{D}$
}

\begin{abstract}
A 28-year-old man presented with a sudden onset of left leg pain and swelling. There was no history of preceding trauma. Initial blood tests were unremarkable, ultrasound imaging showed extensive subcutaneous oedema. He then developed similar symptoms in his right leg, followed by both arms, and finally his forehead. A fluctuating peripheral eosinophilia subsequently developed. Biopsies of the arm and forehead showed an evolving panniculitis. The clinical features, peripheral eosinophilia, imaging and histology were consistent with a diagnosis of eosinophilic fasciitis. The patient received a course of steroids with complete resolution of his symptoms. Eosinophilic fasciitis is an uncommon condition but one that may be encountered by acute physicians. Early recognition and treatment is important to prevent complications.
\end{abstract}

KEYWORDS: Eosinophilic fasciitis, eosinophilia, swelling

\section{Case presentation}

A 28-year-old man presented to the emergency department with sudden onset severe left calf pain while walking. There was no history of trauma or recent strenuous exercise. He was afebrile and vital signs were within normal limits. His left calf was swollen, erythematous and tender. Compartment pressures, pulses and capillary refill time were normal. Limb examination revealed normal power, reflexes and sensation.

He had no medical comorbidities. A year earlier, he had sustained a left common peroneal nerve injury. Three months before presentation, he received antibiotics for an infected neuropathic ulcer on the left foot. As a child, he underwent surgical repair of left tibia and fibula fractures and a left anterior cruciate ligament rupture.

Ultrasound revealed marked subcutaneous oedema, but a normal knee joint and no deep-vein thrombosis. He was admitted to hospital for analgesia. A day after presentation, the pain intensified and localised to his left knee. Magnetic resonance imaging (MRI; Fig 1a) demonstrated extensive subcutaneous oedema from the thigh to the ankle, particularly centred around

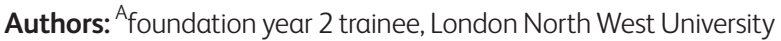
Healthcare NHS Trust, London, UK; ${ }^{B}$ foundation year 2 trainee, London North West University Healthcare NHS Trust, London, UK; ${ }^{C}$ Clinical research fellow, Imperial College London, London, UK; ' ${ }^{\text {consultant }}$ geriatrician, Imperial College Healthcare NHS Trust, London, UK the soft tissues of the knee. A positron emission tomographycomputed tomography (PET-CT; Fig 1b) showed increased tracer uptake in the same area with enlarged inguinal lymph nodes. A biopsy of the left knee demonstrated haemorrhage and perieccrine fat necrosis. A second deep-tissue biopsy was unremarkable.

He was treated with teicoplanin and ciprofloxacin for presumed cellulitis, without effect. Twenty-one days after presentation, he developed pain and swelling in his right calf and knee. Two days later, both hands and his left elbow were affected (Fig 1c). MRI of the left elbow again showed subcutaneous oedema (Fig 1d), and biopsy of his arm was suggestive of an erythema nodosumlike evolving panniculitis. A week later, he developed multiple indurated erythematous lesions over his forehead. A further biopsy revealed fibrosis in the deeper dermis extending to the subcutaneous septa and scattered eosinophils. Throughout this time, there was fluctuating peripheral eosinophilia, with values ranging from $0.0-1.2 \mathrm{~mm} / \mathrm{hr}$

In summary, the key features were multiple areas of skin swelling and pain which were migratory. Initial biopsies of the affected leg showed fat necrosis, but no evidence of specific vasculitis or panniculitis. The clinical condition progressed despite treatment with analgesia and antibiotics, and subsequent imaging and biopsies were arranged. These revealed localised fasciitis. There was peripheral eosinophilia, with fluctuating inflammatory markers.

After discussion within a multidisciplinary team of internal physicians, dermatologists, rheumatologists, radiologists and histopathologists, it was agreed that these features met criteria for a diagnosis of eosinophilic fasciitis.

Methylprednisolone was given followed by oral prednisolone. Within 4 weeks, his symptoms and signs had resolved completely and he was discharged from hospital.

\section{Discussion}

Eosinophilic fasciitis is a rare connective tissue disease of probable autoimmune aetiology. It is characterised by areas of painful swelling and induration in the distal limbs and is associated with characteristic histological findings of fascial thickening and lymphocyte aggregation. ${ }^{2}$ Peripheral eosinophilia is common, but not required for diagnosis, and histology may not show evidence of eosinophils. ${ }^{1}$ There is frequently a history of strenuous activity prior to onset but the time course in this case is longer than typically described. ${ }^{3}$ Late complications can include joint contractures and significant disability, necessitating early 


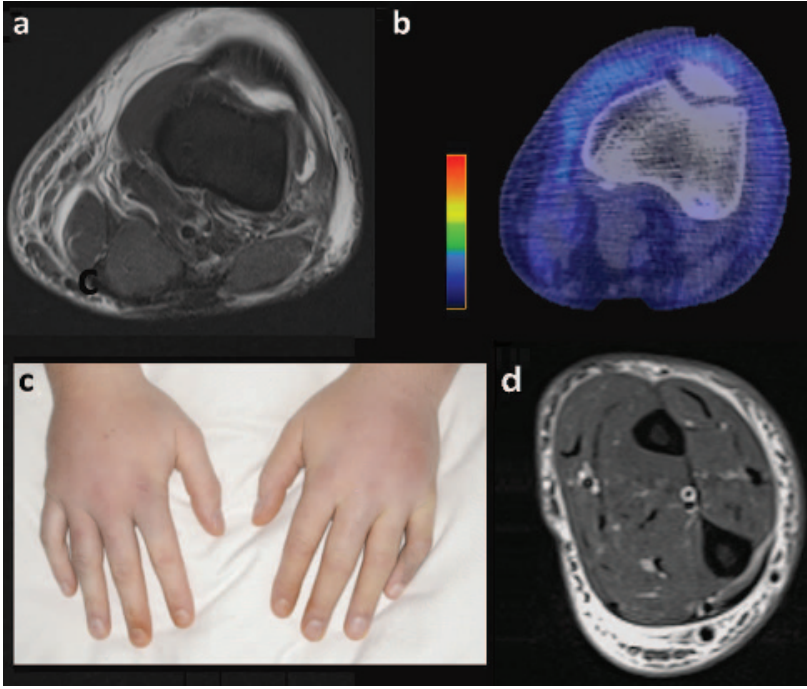

Fig 1. Imaging of panniculitis at multiple sites. a) Magnetic resonance imaging (MRI) of the left leg on presentation which showed diffuse subcutaneous oedema from thigh to ankle. b) Positron emission tomographycomputed tomography showing increased uptake in the soft tissues surrounding the left knee with reactive inguinal lymph nodes. c) Photograph of the bilateral hand swelling. d) MRI of the left elbow showing evidence of localised panniculitis.

recognition and treatment. Steroid treatment is the mainstay of therapy, with some centres combining steroid sparing agents such as methotrexate. ${ }^{1,2}$

A recent case series found that $79 \%$ of cases of eosinophilic fasciitis were initially misdiagnosed, and mean time of onset to diagnosis was 11 months. ${ }^{3}$ Reasons for this include the fact that it is a rare condition, and shares overlapping features with other conditions such as cellulitis. Additionally, peripheral eosinophilia is not always present. Thus, physician awareness and consultation with affiliated specialties, such as radiology and pathology, are essential.

Several forms of cognitive bias were apparent in this case, leading to errors of diagnostic strategy; the peripheral eosinophil count was normal on admission, lowering suspicion for the condition, and aiding confirmation bias for more common explanations of limb pain and swelling; the initial biopsies were non-specific, leading to an anchoring bias towards a diagnosis of trauma or non-specific inflammation; and physician awareness of the condition is low. As a result, the availability heuristic was employed and commoner conditions such as cellulitis and deep-vein thrombosis were considered more readily. Physician awareness of the condition was sufficiently low that factitious causes were considered. This led to diagnostic momentum bias, and possibly delayed the arrival at the correct diagnosis.
In order to counter the cognitive biases described above, a multidisciplinary meeting was arranged to establish the diagnosis. As the condition evolved, further biopsies were obtained which ultimately supported the diagnosis. Additionally, multimodal imaging was critical in supporting the diagnosis, particularly in the early stages when biopsies were non-diagnostic. MRI is the modality of choice but the non-invasive nature and accessibility of ultrasound is increasingly recognised. ${ }^{4}$ PET-CT has been used rarely to identify or exclude systemic disease and identify a suitable biopsy site. ${ }^{5}$ The use of all three modalities to confirm a severe underlying panniculitis was important to focus the diagnosis to the connective tissues.

In summary, eosinophilic fasciitis is an uncommon disease with substantial morbidity and complications, but diagnosis can be challenging. If it is suspected, multimodal investigation and multidisciplinary expertise should be rigorously sought.

\section{Key points}

Clinicians should consider eosinophilic fasciitis in patients presenting with unexplained skin pain and swelling; peripheral eosinophilia may be present in half of cases.

$>$ When diagnosis is uncertain and the patient's clinical condition does not improve, a wide range of multidisciplinary specialists can assist with diagnosis and management.

> Repeating investigations as the clinical condition evolves can reveal the underlying diagnosis.

\section{References}

1 Pinal-Fernandez I, Selva-O' Callaghan A, Grau JM. Diagnosis and classification of eosinophilic fasciitis. Autoimmun Rev 2014:13:379-82.

2 Ihn $\mathrm{H}$. Eosinophilic fasciitis: From pathophysiology to treatment. Allergol Int 2019;68:437-9.

3 Wright NA, Mazori DR, Patel M et al. Epidemiology and treatment of eosinophilic fasciitis: an analysis of 63 patients from 3 tertiary care centers. JAMA Dermatol 2016;152:97-9.

4 Pousa-Martinez M, Ginarte M, Suarez-Penaranda JM, Vazquez-Veiga $\mathrm{H}$. Skin ultrasound is a useful tool for evaluating and monitoring eosinophilic fasciitis. Int J Dermatol 2018;57:743-5.

$5 \mathrm{Kim} \mathrm{HJ}$, Lee SW, Kim GJ, Lee JH. Usefulness of FDG PET/CT in the diagnosis of eosinophilic fasciitis. Clin Nucl Med 2014;39:801-2.

Address for correspondence: Dr Melanie Dani, Department of Geriatric Medicine,Imperial College Healthcare NHS Trust, Hammersmith Hospital, Du Cane Road, London W12 OHS, UK. Email: melanie.dani@nhs.net 